


\title{
Mecanismos lingüísticos de atenuación en conversaciones coloquiales españolas y alemanas $^{1}$
}

\author{
Mitigation strategies in Spanish and German colloquial \\ conversations
}

\author{
Josefa Contreras Fernández \\ Universitat PolitèCNIA De VALĖNCIA \\ ESPAÑA \\ jcontre@idm.upv.es
}

Recibido: 20-II-2020 / Aceptado: 21-IX-2020

DOI: $10.4067 /$ S0718-09342020000300885

\section{Resumen}

Existe una gran variedad de estudios sobre la atenuación lingüística en diferentes géneros y registros, pero no son tantos los trabajos dedicados a la comparación interlingüística e intercultural. El presente trabajo se centra en la comparación de este fenómeno pragmático en dos lenguas, español peninsular y alemán. Se pretende conocer qué mecanismos lingüísticos utilizan los hablantes nativos en conversaciones coloquiales en ambas lenguas, si hay similitudes o diferencias en las tácticas ${ }^{2}$ lingüísticas utilizadas en estas conversaciones y a qué se deben. Para ello se parte de una visión pragmática de la atenuación y de una perspectiva teórica de naturaleza intercultural y sociocultural con el fin de poder explicar las razones de los resultados obtenidos de nuestro corpus. Este está formado por nueve conversaciones, pertenecientes al corpus Val.Es.Co., y cinco correspondientes al Datenbank für gesprochenes Deutsch ('banco de datos para el alemán hablado') con los mismos minutos analizados y contabilizados manualmente. Esta metodología era imprescindible para decidir si una táctica lingüística funcionaba como mecanismo de atenuación o no. Un primer resultado muestra que hay mecanismos que se emplean en una lengua para atenuar pero no en la otra, por razones de diversidad tipológica lingüística, como es esperable. Lo interesante de este resultado es observar qué tácticas emplea, a cambio, la otra lengua. Un segundo resultado es que el grupo de mecanismos que son comunes en ambas lenguas difiere en frecuencia por causas socioculturales, como es la forma de actuar más extrovertida y abierta de los españoles frente a una interrelación con predominio del respeto por el territorio de uno mismo y del otro. 
Palabras Clave: Mecanismos lingüísticos de atenuación, conversaciones coloquiales, análisis contrastivo español-alemán, contexto sociocultural.

\begin{abstract}
There is a great variety of studies on linguistic mitigation in different genres and registers, but there are not so many research into interlinguistic and intercultural comparison. This paper focuses on the comparison of this pragmatic phenomenon in two languages, Peninsular Spanish and German. The purpose is to find out what mechanisms native speakers use in colloquial conversations in both languages, if there are similarities or differences in the mechanisms used in these conversations and what they are due to. This is based on a pragmatic vision of mitigation and on an intercultural and sociocultural theoretical perspective, in order to explain the reasons for the results obtained. Our corpus consists of nine conversations belonging to the Val.Es.Co. corpus and five corresponding to the Datenbank für gesprochenes Deutsch ('data bank for spoken German') with the same minutes analyzed and manually counted. This methodology was essential to decide whether a mechanism worked as an mitigation mechanism or not. A first result shows, as expected, that there are mechanisms that are used in one language to mitigate but not in the other, for reasons of linguistic typological diversity. This finding is interesting because it reveals which mechanisms the other language uses instead. A second result is that the group of mechanisms that are common in both languages differs in frequency due to socio-cultural causes, such as the more outgoing and open nature of the Spanish speakers in contrast to the interactions which are more respectful of one's own territory and of the other, characteristic of the German culture.
\end{abstract}

Key Words: Mitigation mechanisms, colloquial conversations, Spanish-German contrastive analysis, sociocultural context.

\title{
INTRODUCCIÓN
}

La atenuación lingüística ha sido muy desarrollada en los últimos años, en particular a partir del estudio de diversos mecanismos lingüísticos, Albelda, (2018), Uclés Ramada (2018), Soler Bonafont (2018) y de diversas manifestaciones según los géneros y los registros, es decir, más formales Figueras (2017), Kotwica (2018), Villalba (2018) y más informales como Samper-Hernández (2018). Asimismo, existen estudios contrastivos español-alemán sobre mecanismos lingüísticos de atenuación en tertulias televisivas (Contreras, 2017), en correos electrónicos (Contreras \& Zhao 2017) y en géneros literarios (Contreras, 2018), sin embargo, falta un estudio contrastivo español- alemán en el registro coloquial y en el género de la conversación espontánea. Así, el objetivo de esta investigación es averiguar qué mecanismos lingüísticos utilizan los hablantes nativos en conversaciones coloquiales, si hay similitudes o diferencias en los mecanismos utilizados en estas conversaciones donde la relación es diferente que en los discursos anteriores, y, si existen, a qué se pueden deber esas similitudes o diferencias.

En los estudios contrastivos antes mencionados, se pudo observar que en las tertulias televisivas los hablantes alemanes utilizaban, sobre todo, el Konjunktiv II (condicional) $y$, en las españolas, los interlocutores, si bien hacían uso de 
modalizadores de tiempos verbales del condicional y el imperfecto, empleaban con más frecuencia modificadores morfológicos internos (diminutivos, habitualmente) para atenuar su discurso (Contreras, 2017). En las conversaciones de los correos electrónicos (Contreras \& Zhao, 2017), que pertenecen a un género híbrido, las tácticas más utilizadas por los estudiantes alemanes fueron también el Konjunktiv II (condicional), partículas y adverbios modales y construcciones justificadoras. Estas últimas tácticas lingüísticas de atenuación fueron las más utilizadas en los correos electrónicos españoles, además de los modalizadores de tiempos verbales, en concreto, el condicional y el imperfecto. En los géneros más formales (Contreras, 2018) destacó, sobre todo, un porcentaje elevado de construcciones impersonales.

A partir de estos estudios previos, se plantea si es de esperar que los mismos mecanismos de atenuación empleados en otros géneros se encuentren en las conversaciones coloquiales o si, por el contrario, se puede esperar que haya diferente uso debido a la naturaleza de las conversaciones coloquiales. Asimismo, dado que la cultura alemana es una cultura de más distanciamiento que la española (Haverkate, 2004; Briz, 2007) o de más privacidad (Contreras, 2004, 2012), se parte de la hipótesis de que en el corpus alemán se registrarán más mecanismos de atenuación que en el español.

Con el fin de lograr los objetivos del presente estudio, se ha estructurado este trabajo de la siguiente manera: se presentará el marco teórico, el corpus y la metodología que contextualiza este trabajo, seguidos de los resultados cualitativos y cuantitativos para poder explicar, en el apartado de la discusión, los datos obtenidos en esta investigación.

\section{Marco teórico}

Este apartado consta de tres subapartados, en concreto, la imagen, la atenuación y la incidencia de los factores culturales en los fenómenos pragmáticos, dentro de los cuales se incluyen investigaciones anteriores y enfoques teóricos que consideramos relevante para la contextualización de este estudio.

\subsection{Imagen}

Goffman (1967) fue el primero en occidente en desarrollar el concepto de imagen social. Según este autor toda persona tiene unas necesidades de imagen en sus relaciones interpersonales y, con el fin de que las actuaciones de una persona estén en consonancia con su imagen social, este sociólogo propone unas acciones que salvaguarden, protejan y reparen la imagen. Posteriormente, Brown y Levinson (1978, 1987), Lakoff (1972) y Bravo (1993), entre otros, basaron sus postulados sobre la cortesía en este concepto de imagen. Para Brown y Levinson $(1978,1987)$ la imagen siempre se ve amenazada durante las interacciones, por lo que establecieron unas 
estrategias de cortesía o actividades de imagen con el fin de protegerla. Según Hernández Flores (2008, 2013), el concepto de actividad de imagen, sin embargo, no siempre se relaciona con la cortesía, sino también con la descortesía (Bernal, 2007; Kaul de Marlangeon, 2008) o con la propia imagen (Boretti, 2005, Sifianou, 2012, Hernández Flores, 2013; Albelda, 2016). En el presente trabajo se analizarán y ejemplificarán mecanismos de atenuación que tienen como fin salvaguardar tanto la propia imagen como las de los demás, así como proteger o reparar la imagen ante posibles daños o amenazas.

\subsection{Atenuación}

La atenuación es una categoría pragmática que se emplea con el fin de expresar lo dicho de una forma más vaga o imprecisa (Albelda, Briz, Cestero, Kotwica \& Villaba, 2014). Los atenuantes, también considerados hedges (Lakoff, 1972), son mecanismos para reducir la fuerza ilocutiva de un acto de habla (Fraser, 1980; Bravo, 1993; Briz, 1995, 2003, 2007; Caffi, 1999, 2007; Albelda, 2008; Contreras, 2012; Mihatsch, 2013; Cestero \& Albelda, 2012; Albelda et al., 2014; Albelda, 2016, Contreras, 2018). La atenuación tiene como función mitigar, reparar o proteger $y$, a su vez, está relacionada con la preocupación por la propia imagen y la necesidad de evitar la responsabilidad sobre lo dicho o hecho. Albelda (2016) define la atenuación como sigue:

"La atenuación es una estrategia pragmática (comunicativa) originada por necesidades de imagen y dirigida a mitigar y minimizar la intensidad de lo que se expresa reduciendo la fuerza ilocutiva del acto de habla, y en ocasiones, a través de mecanismos de lenguaje vago por lo que se difumina o minimiza el contenido proposicional. La atenuación es, al mismo tiempo, una actividad argumentativa que permite a los hablantes formular un menor compromiso hacia lo dicho y así lograr más eficazmente las metas conversacionales de los participantes en el discurso" (Albelda, 2016: 30).

Los mecanismos de atenuación son recursos lingüísticos que se utilizan para atenuar lo dicho o el decir. Estos mecanismos pueden ser morfológicos, como los diminutivos, o léxicos, como los modificadores externos o las partículas discursivas de evidencialidad, entre otros, o recursos paraverbales como la risa (Bravo, 1993; Contreras \& Zhao, 2017). Asimismo, pueden ser estructuras más complejas y afectar al contenido proposicional del enunciado o pueden también ser extraproposicionales y afectar a la enunciación.

Para Briz y Albelda (2013) es imprescindible fijarse en la función del atenuante con el fin de poder entender por qué se utilizó una táctica lingüística de atenuación. Según estos autores, la imagen siempre está involucrada, pero de tres formas distintas, a saber, autoprotección, prevención y reparación ${ }^{3}$. La función de autoprotección es la referente a la de salvaguarda del yo o "velar por sí mismo autoprotegiéndose por lo dicho o por lo hecho, con un interés de ganar o no perder la imagen" (Albelda et al., 
2014: 26), siendo algunas de las funciones más específicas que se incluyen dentro de esta:

“evitar responsabilidades de lo dicho que pueda afectar a la propia imagen; autoprotegerse generalizando o expresando lo dicho como una evidencia, o preocupación por el qué dirán los demás.” (Albelda et al., 2014: 26).

La función de prevención hace referencia a un posible daño hacia otra persona, cuando se puede ver amenazada la imagen de esa persona o su territorio, anticipándose a lo que podría ser un problema o previniendo conflictos. La función reparativa consiste en reparar una amenaza a la imagen del otro y tiene lugar cuando ya se ha producido el daño (Albelda et al., 2014). Dado que este trabajo se enmarca dentro de la pragmalingüística y la sociopragmática, se presentan a continuación algunos aspectos culturales relacionados con las comunidades de habla objeto de este estudio.

\subsection{La incidencia de los factores culturales en los fenómenos pragmáticos}

Como señalan Scollon y Scollon (1995), en cada cultura los hablantes tienen interiorizadas ciertas costumbres y formas de actuar que se manifiestan en el quehacer cotidiano y, por tanto, influyen en las interacciones y en el comportamiento de los hablantes durante las mismas. Por ello, es importante contar con la influencia de los factores culturales para analizar los hechos lingüísticos en estudios interculturales. Siguiendo este criterio, se ha de tener en cuenta el contexto sociocultural de la cultura alemana y el de la española peninsular en el análisis de los mecanismos de atenuación.

En Contreras (2012) se observó que la cultura alemana, según Althaus y Mog (1996), le otorga una gran importancia al tiempo libre y que esto, a su vez, se relaciona con la separación de lo privado (por ejemplo, el tiempo libre) de lo ajeno o público (por ejemplo, el trabajo). En España, en cambio, la importancia recae más sobre la familia. Los españoles se caracterizan por su forma de actuar más extrovertida y abierta (Bravo, 1993; De Miguel, 1997) y por la importancia que le otorgan a las amistades.

"El hecho de tener muchos amigos se considera un mérito, una gracia especial, en una sociedad, como la española, donde se vive tanto hacia fuera." (De Miguel, 1997: 39-40).

Estas características socioculturales también se reflejan en las conversaciones: en las alemanas los interlocutores mantienen entre sí una mayor distancia física que los españoles, además suele haber más interrupciones y menos silencios en los discursos españoles (Contreras, 2008). En este sentido, se ha caracterizado la cultura española como una cultura de más confianza (Bravo, 1993; Hernández-Flores, 2003) y la 
alemana como una cultura de más privacidad (Contreras, 2004, 2008). En palabras de Scollon y Scollon (1995), en la cultura española predominan más las ingroup-relationships mientras que en la cultura alemana se diferencia entre las ingroup-outgroup relationships.

\section{Corpus y metodología}

Las conversaciones han sido seleccionadas teniendo en cuenta el género discursivo y el registro, en nuestro caso, el género de conversación espontánea relacionado con un registro coloquial.

Se ha trabajado con nueve conversaciones españolas pertenecientes al corpus Val.Es.Co., en concreto, al Corpus de conversaciones coloquiales (Briz \& Grupo Val.Es.Co. (Valencia Español Coloquial) (2002) y al Corpus Val.Es.Co 2.1 (2019), que suman un total de 184 minutos. Las conversaciones alemanas son cinco y suman en total 182 minutos, correspondientes al corpus Datenbank für gesprochenes Deutsch ('banco de datos para el alemán hablado'), en concreto, al corpus FOLK, dentro del cual están, entre otras conversaciones, las conversaciones coloquiales ('Alltaggepräche). El objetivo era conseguir los mismos minutos para poder contrastar mejor las tácticas lingüísticas de atenuación utilizadas por los hablantes de ambos corpus. En el corpus alemán hay menos conversaciones debido a la longitud de las mismas, la mayoría son de veinte o treinta minutos, incluso hay una conversación, la E-00042, que es de una hora.

El nivel de instrucción de los hablantes es alto, de diferentes profesiones ${ }^{4}$, muchos de los interlocutores de ambos corpus son estudiantes y la relación que hay entre ellos es de proximidad, pues son conocidos, compañeros de clase, amigos, e incluso parientes. Criterios, que según Briz y Grupo Val.Es.Co. (2002: 19) caracterizan las:

"conversaciones coloquiales prototípicas, pues presentan una mayor relación de igualdad social y funcional entre los interlocutores, una mayor relación vivencial de proximidad y una temática no especializada."

Asimismo, se han elegido estas conversaciones considerando la temática tratada en las mismas. Tanto en el corpus alemán como en el español los temas son cotidianos, versan sobre la política, la homosexualidad, el alcohol, las drogas, los exámenes, las comidas, la casa, el trabajo, el cine y los viajes. Derivado de ello, se puede concluir que en ambos corpus, el fin de las interacciones es interpersonal.

En cuanto a la identificación de mecanismos lingüísticos que puedan funcionar como recursos de atenuación, tanto para el análisis español como para el alemán, se ha partido de la propuesta de Albelda et al. (2014) 5 . Para el caso del alemán, puesto que la propuesta de partida se centra en el español, se adaptó dicha ficha, pero incluyendo la táctica lingüística a través de partículas modales, ya que como se explicará en el siguiente apartado, estas no existen en la lengua española. De acuerdo con este estudio, para decidir si una táctica funciona como mecanismo de atenuación, es importante identificar, por una parte, el contexto interactivo concreto, es decir 
factores situacionales como la temática tratada o la relación entre los interlocutores, y los rasgos de los hablantes y, por otra parte, el desencadenante con el propósito de confirmar si el recurso lingüístico utilizado tiene un valor atenuado. Así, el análisis de los mecanismos lingüísticos de atenuación en las conversaciones de ambas lenguas se realizó cualitativamente. Posteriormente se cuantificaron los recursos lingüísticos tomando en consideración las tácticas lingüísticas de atenuación de la ficha propuesta por Albelda et al. (2014) tanto para el corpus español como para el alemán. En lo referente a las funciones de la atenuación, mencionadas en el marco teórico, era imperativo que la imagen estuviera implicada, bien con la función de autoprotección, prevención o reparación. La imagen está implicada, si se ha hecho uso de un distanciamiento del mensaje con el fin de evitar responsabilidad sobre lo afirmado o para evitar algún tipo de desacuerdo o, incluso, para reparar algún posible daño a la imagen del otro.

A continuación se presentan los resultados de esta investigación. En el apartado tres se presentan los resultados cualitativos y en el apartado 4 los cuantitativos. Se ha optado por la combinación de ambas metodologías con el fin de poder contrastar los mecanismos de atenuación utilizados con las ocurrencias y porcentajes de aparición en cada corpus, el español y el alemán.

\section{Resultado cualitativo}

En este apartado se analizan los mecanismos de atenuación más utilizados en cada corpus y se concluye con las tácticas lingüísticas que han sido utilizadas de forma diferente o que no han sido empleadas por los hablantes del otro corpus. En primer lugar, se ejemplifican los mecanismos de atenuación pertenecientes al corpus español y, a continuación, se tratarán los correspondientes al corpus alemán. En los ejemplos se marcará en negrita solamente el mecanismo de atenuación que se esté explicando, pero no otros que puedan aparecer también en el mismo fragmento, con el fin de apreciar mejor la táctica en cuestión, importante, sobre todo, para los ejemplos alemanes.

Las construcciones justificadoras han sido los mecanismos más utilizados en el corpus español con 143 ocurrencias. Las justificaciones tienen la función de asegurar la credibilidad de lo que se argumenta.

En el ejemplo (1) L y E son dos amigas que están hablando sobre diversos temas

en la casa de E. L utiliza una orden, un acto directivo en beneficio de E y, por consiguiente, una posible amenaza para la imagen de L. 
(1)

L: que me he acordado que me tienes que dar los apuntes $\$$

E:

L: y me los das ahora $\uparrow / /$ y así me los lle[vo yo]

$\mathrm{E}$ :

[sí]

Siay! sí sí

(Briz et al. 2002: Corpus de conversaciones Val.Es.Co, L.15.A, líneas 202-206)

El objetivo de emplear este recurso de justificación es para mitigar la fuerza ilocutiva del acto directivo y alcanzar el propósito argumentativo, protegiendo tanto la propia imagen de L como la de E. Este acto directivo podría desencadenar en un daño a la relación de amistad, L lo atenúa con el fin de salvaguardar tanto su propia imagen como la de la amiga.

La atenuación a través de partículas discursivas y expresiones de control de contacto es el segundo mecanismo más utilizada en el corpus español con 84 ocurrencias. Según Uclés Ramada (2018), las partículas fático-discursivas ‘¿eh?’ y '¿no?', son partículas asociadas más al discurso oral que al escrito (Albelda, 2016). A través de estos marcadores conversacionales, o partículas fático-discursivas, se busca el consentimiento y la alianza con el interlocutor, a la vez, que se minimiza un posible desacuerdo con el otro (Uclés Ramada, 2018). Briz (1998) categoriza estas partículas dentro de las tácticas de atenuación y en Contreras (2004) se advierte la función afiliativa de ‘¿eh?’ y ‘'no?' en las conversaciones españolas tanto coloquial como transaccional, pues estos marcadores fático-apelativos son mecanismos de atenuación, a través de los cuales se pretende un consentimiento del otro o una alianza con él.

En el ejemplo (2) el contexto es el siguiente: $\mathrm{E}$ ha estudiado en un colegio concertado de curas. G busca, a través de este marcador fático-discursivo, la alianza con $\mathrm{E}$, a la vez, que atenúa un posible daño a la imagen de esta.

(2)

G: curas asquerosos ¿no?

E: bueno uno- noo

G: no $\downarrow$ hay ge- gente que se puede soportar y gente que no// y allí pues habrá de todo ¿no?

(Briz et al. 2002: Corpus de conversaciones Val.Es.Co, L.15.A, líneas 89-92)

E no consigue su meta discursiva, es decir, la alianza con E, ya que E no está de acuerdo con él. Por ello, responde utilizando varios recursos de atenuación para proteger su imagen y reparar el posible daño causado a E. Además de la partícula fático-discursiva, G utiliza una construcción impersonal generalizando su enunciación. Según Briz (2001) la impersonalidad se identifica con la despersonalización u ocultación del agente y se usa como atenuación tanto en géneros formales como informales (Cestero \& Albelda, 2012; Caffi, 1999, 2007; Luukka \& Markkanen, 1997). 
Esta estrategia de atenuación ha sido muy utilizada por los hablantes de ambos corpus. No obstante, en el corpus español analizado los interlocutores han empleado esta táctica algo diferente que los interlocutores del corpus alemán. En el ejemplo (2) $G$ mitiga su acto de habla asertivo generalizando y en el siguiente fragmento se encubre la opinión en una tercera persona ('mi hijo', 'mi Jose') o se apela a las palabras de otros ('me dicen') con el fin de proteger la propia imagen, desfocalizando, de este modo, el papel de los participantes en la enunciación.

(3)

\section{A: mi hijo me lo explicóo $\downarrow$ \\ C: sí $\downarrow$ me dicen el del pepéy las Prov-/ en esoo $\uparrow[(())$ ((no me oirás))] \\ A: $[(($ yo- dijeron $\uparrow))]$ dijo mi Jose}

(Val.Es.Co 2.1 conversación 25, líneas 44-57)

El contexto es el siguiente. Unas señoras están en un quiosco hablando sobre si el periódico "Las Provincias" está vinculado al partido político del PSOE (Partido Socialista Obrero Español), que es un partido de izquierdas, o al del PP (Partido Popular), que es de derechas. Los interlocutores hacen uso de la táctica lingüística de la impersonalización con el fin de desfocalizar su opinión en la enunciación y proteger, de este modo, su propia imagen.

En lo referente a los modificadores morfológicos internos, el diminutivo se emplea en la lengua española como modificador bien para reducir el tamaño, para expresar afecto o cercanía, o bien como atenuador. Según Albelda y Briz (2010), el diminutivo se define como:

"una categoría pragmática cuya función consiste en minimizar la fuerza ilocutiva de los actos de habla y, con frecuencia, regula la relación interpersonal y social entre los participantes de la enunciación." (Albelda \& Briz, 2010: 238).

Los modificadores morfológicos internos, con 40 ocurrencias en el corpus español, son, a la par con los elementos paralingüísticos, el quinto recurso más utilizado en esta lengua. En el ejemplo (4) unos amigos quedan para merendar en la playa de El Saler, en Valencia, donde también hay algunos árboles, pero la persona que ha elegido el lugar no ha tenido en cuenta que hubiera sombra. El hablante A se lo reprocha a su amigo, pero al hacer uso del diminutivo minimiza la fuerza ilocutiva de su acto de habla y, con ello, una posible amenaza a la imagen de la otra persona.

(4)

A: falta un poqui]llo más de sombra pero vamos $\downarrow$ tampocoo

(Briz et al. 2002: Corpus de conversaciones Val.Es.Co, H.38.A, línea 13) 
Las fórmulas apelativas, con 41 casos analizados en las conversaciones del corpus español, son el cuarto recurso más utilizado. La apelación se construye a través de formas de tratamiento convencionalizadas como 'oye', 'hombre' etc. o por medio de tratamientos nominales o pronominales. Blas (1994) relaciona estas formas de tratamiento con la cortesía, al estar relacionadas con la prevención de una posible amenaza a la imagen del otro. En nuestro corpus español se han contabilizado formas de tratamiento convencionales y nominales como se puede observar en los siguientes ejemplos. En (5) se ejemplifica una de las fórmulas apelativas más utilizada en este corpus, 'hombre', con función atenuadora. El contexto es el siguiente: unos amigos están hablando sobre personas conservadoras o liberales y los interlocutores no comparten la misma opinión:

(5)

E: ¡hombre! Yo- o sea yo por liberal $\uparrow$ no entiendo esto $§$

G: Shombre liberal/ lo que pasa es que no sé pues/ tú a lo mejor entiendes por liberal pues/// ((hay)) gente que entiende $\rightarrow$ una viva la virgen ¿no? o sea// que pasan de todo que que

(Briz et al. 2002: Corpus de conversaciones Val.Es.Co, L.15.A, líneas 391-395)

Los interlocutores utilizan las fórmulas apelativas para manifestar la relación de cercanía y cordialidad que tienen, mitigar la enunciación y prevenir una posible amenaza a la imagen. En el ejemplo (6) se ha utilizado una forma nominal con la misma función:

(6)

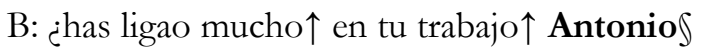

A: \टं y esa chica que conocías?

(Briz et al. 2002: Corpus de conversaciones Val.Es.Co, H.38.A, líneas 72-73)

A través del vocativo, 'Antonio', B minimiza la fuerza ilocutiva de la pregunta tan directa formulada a A. De esta forma, se protegen las imágenes de ambos y se evita un posible conflicto; de hecho, A cambia de tema. Como se verá en los resultados cualitativos del corpus alemán, no se han contabilizado nombres propios como mecanismo de atenuación en las conversaciones analizadas.

En las conversaciones analizadas del corpus alemán, el recurso lingüístico de atenuación más utilizado son las partículas modales, con 276 ocurrencias. Esto se debe, por una parte, a que las partículas modales se emplean, sobre todo, en el discurso oral, y manifestación prototípica de este son las conversaciones coloquiales, objeto de esta investigación, y, por otra parte, al hecho de que, al igual que se pueden utilizar estas partículas con función intensificadora, se usan también con mucha frecuencia en su función atenuadora (Holly, 1979; Langner, 1994; Waltereit, 2006). Muchas de las partículas modales se pueden combinar entre ellas, por lo que o bien 
puede aparecer solamente una o bien varias de ellas en la misma oración, como se observa en el ejemplo (7) de la conversación E-000426.

Como es sabido, las partículas modales alemanas no tienen significado léxico, solo tienen un valor comunicativo ilocutivo, pueden atenuar o intensificar puntos de vistas de los interlocutores o contribuir a un consenso con el otro (Waltereit, 2006) y, de esta forma, respetar el territorio $y$, por consiguiente, prevenir una posible amenaza a la imagen. Para determinar el valor de una partícula modal hay que establecer una oposición entre la oración con partícula y sin partícula. Por ejemplo, en el acto directivo del siguiente fragmento, si se omitieran las partículas modales doch y mal, el enunciado presentaría una amenaza para la imagen del interlocutor.

(7)

LK [hör mir doch mal zu] ${ }^{7}$

LK ich will halt damit nur sagen dass ich die führungspositionen bitte nach leistung besetzt se[hen will]

Escúchame Yo solo quiero decir con eso que quiero ver las posiciones de liderazgo por favor ocupadas según la eficacia].

(Datenbank für gesprochenes Deutsch, FOLK- E-00042, líneas 0103-0201)

En (7) unos amigos están discutiendo sobre la paridad de profesores y profesoras en la universidad y, en concreto, sobre mujeres y hombres en posiciones de poder y LK dice: Escúchame y atenúa este imperativo con dos partículas modales doch mal, y a través de la partícula modal halt el hablante se justifica de alguna forma, sin querer imponer con tanta intensidad su opinión y previene así una posible amenaza a la imagen del otro.

En el fragmento de la conversación E-00300, unas amigas se han juntado para cocinar en la casa donde se aloja $\mathrm{OE}$ y esta quiere abrir la ventana.

(8)

OE machen wir vielleicht ma_s fenster auf [oder]

DB [aber_s is] voll kalt lass uns lieber schwitzen orea

OE... nee ich mein nur sons stinkt dann die ganze wohnung

$O E:$ Abrimos la ventana, no

DB: pero hace mucho frío, deja que mejor sudemos

OE no, yo creo solo que sino apesta todo el piso

(Datenbank für gesprochenes Deutsch, FOLK- E-00300, líneas 1062-1073)

OE reduce la fuerza ilocutiva del acto de habla directivo a través de las partículas modales vielleicht mal, una expresión de control de contacto, oder ('o' / 'no'), y una justificación con el fin de prevenir un posible desacuerdo o rechazo. 
Las construcciones de justificación son los mecanismos de atenuación más utilizados, después de las partículas modales, con un resultado de 139 ocurrencias. En (9) unas amigas están hablando en la cafetería de la universidad sobre los exámenes y sobre el hecho de acudir a una tutoría:

(9)

AM wann warst du in der sprechstunde lena (.)

$\mathrm{LP} \operatorname{mh}(0.31)$

AM ((Schnalzlaut)) wann warst du in der sprechstunde

LP (.) is schon länger her (0.72) also s_war ganz spontan weil ähm (.) ich hatt des nich geplant (0.9) und äh weil ich wollt mit dir ja eigentlich wir wollten ja zusammen [hin]

AM: Cuándo estuviste en la tutoría, Lena

LP: mh (silencio de 0,31)

AM: (hace sonido para que LP conteste) cuándo estuviste en la tutoría

LP: ya hace tiempo (silencio) bueno, fue muy espontáneamente, porque no lo tenía planeado (silencio) y porque yo quería en verdad queríamos ir juntas

(Datenbank für gesprochenes Deutsch, FOLK- E-00046, líneas 0052-0062

Con el fin de proteger su imagen y reparar el posible daño a la imagen del interlocutor (AM), LP utiliza, además de las construcciones justificadoras (is schon länger her (0.72) also s_war ganz spontan weil ähm (.) ich hatt des nich geplant (0.9) und äh weil ich wollt mit dir ja eigentlich wir wollten ja zusammen [hin]), otros recursos, dentro de esta justificación, como la partícula modal ja, el evidencial eigentlich ('en verdad') y, además, una parcelación sintáctica, que crea una mayor distancia hacia lo dicho al ir proporcionando la información poco a poco.

Las construcciones acotadoras de la opinión es la cuarta táctica más utilizada en el corpus alemán con 80 ocurrencias y con una gran variedad de expresiones empleadas por los interlocutores (glaube ich, finde ich, mein ich, meiner Meinung nach, in meinen Augen, in meinem Denken, etc.). Este recurso se utiliza para restringir la opinión y, de esta forma, mostrar que solamente se trata de la opinión del hablante, respetando así la opinión de los demás, como se observa en el ejemplo (10), donde unos amigos están hablando del hecho de que cuando se bebe alcohol, uno ya no controla. Sin embargo, AM es de la opinión (bin ich der meinung) que cuando se tiene un poco de entrenamiento sí que se puede controlar

AM also wenn en bissl training hat dann kann man des schon kontrollieren bin ich der meinung

(Datenbank für gesprochenes Deutsch, FOLK- E-00042, línea 1304) 
En (11) este mismo recurso lingüístico cumple la función de hacer menos impositiva la sugerencia. En la conversación E-00300 unas amigas están preparando una comida a la que le añaden bastante mantequilla, pero TP cree (glaub $i c h)$ que para nada hay que poner tanta.

TP... so viel muss man glaub ich gar nich reinmachen

(Datenbank für gesprochenes Deutsch, FOLK- E-00300, línea 0411)

De este modo el hablante presenta su propia opinión, pero al usar 'creo yo' la presenta acotada al terreno de él mismo con el fin de respetar las opiniones de las demás y evitar una posible amenaza a la imagen.

Como anteriormente se ha comentado, en el corpus alemán no se utilizan modificadores morfológicos internos con función atenuadora, pero sí modificadores morfológicos externos, de los que se han contabilizado 98 ocurrencias en las conversaciones alemanas. Los modificadores morfológicos externos son expresiones que debilitan el significado de lo dicho, como se observa en el ejemplo (12). DM está en la casa de unos amigos narrando un viaje que hizo a Naumburg (Alemania) y cuenta lo que le ocurrió cuando llegó a Großjena empapado y se extravió un poco (en bisschen) en las granjas de ese pueblo

DM:so kam ich denn auch nach $\rightarrow$ // Großjena $\rightarrow$ / wo ich dann schon völlig $\rightarrow$ / durchgeweicht war $\rightarrow /$ und $\rightarrow /$ hab mich dann auch en bisschen verirrt in den Gehöften $\downarrow /$

(Datenbank für gesprochenes Deutsch, BR 001, línea 300)

A través de este recurso, el hablante DM minimiza el significado del verbo (verirren / 'extraviarse') y con ello la enunciación y, de esta forma, protege su propia imagen.

En cuanto a las fórmulas apelativas, se han contabilizado solamente 6 casos en las conversaciones alemanas, frente a las 41 ocurrencias en el corpus español. En el corpus alemán los interlocutores no han utilizado nombres propios como en el corpus español, solamente fórmulas apelativas convencionalizadas como 'hombre' (mensch) y a través de la interjección ey que se podría traducir por las partículas discursivas 'oye' o 'venga', como se observa en (13):

LK ich muss des mit den fingern essen ey

LP mit den fingern essen

LK ja

LK kannste net so essen

$L K$ tengo que comer esto con los dedos, oye 


\section{LP comer con los dedos}

\section{$L K s i$}

\section{LP no puedes comer asi}

(Datenbank für gesprochenes Deutsch, FOLK- E-00042, líneas 0709-0714)

En este fragmento unos amigos están comiendo en la cafetería universitaria y LK utiliza una fórmula apelativa como mecanismo lingüístico para atenuar y salvaguardar su propia imagen. LK busca, a través de este mecanismo de atenuación, justificar y atenuar la enunciación, el hecho de comer con los dedos.

En el corpus alemán se han contabilizado 66 casos de construcciones impersonales atenuantes, frente a las 46 ocurrencias en el corpus español. Pero en lo referente a esta táctica de atenuación hay una diferencia notable si nos fijamos en los porcentajes de la tabla 1 del siguiente apartado. El resultado es más alto en el corpus español, 8,81\%, frente a un 6,85\% de esta táctica usada en las conversaciones alemanas. Esta variación que se observa es debido a que se han tomado para el cálculo de los porcentajes, los totales de los mecanismos de atenuación utilizados en cada conversación, a saber, 963 en las conversaciones alemanas y 522 registradas en el corpus español. Asimismo, los recursos empleados en el corpus alemán también difieren. En las conversaciones alemanas se ha hecho uso, sobre todo, de los pronombres indefinidos man y jemand utilizados de forma substantivada, como se advierte en los siguientes ejemplos. En la conversación E-0046 se utiliza este atenuante con el fin de mitigar la fuerza ilocutiva del acto de habla y difuminar la enunciación, como se puede apreciar en (14):

AM ich [du brauchst zwei semester um (dich in germanistik) prüfen]

LP [weischt ich hab des auch total zufällig mitgekriegt]

AM (.) (aber) [leo man muss sich doch irgendann drum] kümmern mi mich macht des total verrückt in den let[zten zwei wochen]

VW [nee noch schlimmer als stadt_c]

LP [ich mach überhaupt gar] keinen str[ess]

VW [ja] auf jeden fall man muss sich dr[um kümmern weil ich wollte des]

AM [der leo der macht des al]les zwei wochen vorher und dann sieht er dass er dass er sich viel zu sp[ät drum gekümmert hat]

AM: yo [tú necesitas dos cuatrimestre para poder presentarte al examen en germanística

LP lo sé me he enterado totalmente por casualidad

AM (pero) [leo hay que ocuparse en algún momento de ello a mi también me ha vuelto loca en las dos últimas semanas]

$V W[$ no aun pero que la ciudad c]

\section{LP [yo tampoco me estreso para nada]}

$V W$ [si] en cualquier caso hay que ocuparse de [eso porque yo quería]

AM [el leo lo hace 2 semanas antes y luego ve que se ha ocupado de eso demasiado tarde]

(Datenbank für gesprochenes Deutsch, FOLK- E-00046, líneas 0602-0622) 
En (15) DM generaliza para despersonalizar a través del pronombre indefinido jemand ('alguien') en vez de emplear $i b r$ ('vosotros') con el fin de atenuar una posible amenaza a la imagen de los interlocutores, puesto que la novela de Siddharta del escritor alemán Hermann Hesse se supone que es conocida por la mayoría de los alemanes con estudios universitarios como es el caso de los amigos que participan en esta conversación.

\section{(15)}

$\mathrm{DM} \ldots$ also $\rightarrow$ / ich mußte an den $\rightarrow$ / SidDHARta denken $\downarrow /$ falls das jemand kennt $\downarrow /$ ja $\rightarrow$ /

DM bueno/ yo tuve que pensar en Siddharta, por si alguien lo conoce

(Datenbank für gesprochenes Deutsch, BR 001, línea 211)

En las conversaciones alemanas se ha utilizado el recurso de la atenuación a través de la impersonalización del sujeto semántico, apelando al juicio de la mayoría o a un interlocutor general mediante los pronombres man y jemand, sobre todo, con la función de prevenir una posible amenaza a la imagen del otro. En cambio, en el corpus español, como se ha señalado previamente, los hablantes han hecho uso de esta táctica lingüística para encubrir la opinión propia en la opinión de otras personas o apelar a las palabras de otros con el fin de proteger la propia imagen, desfocalizando, de este modo, el papel de los participantes en la enunciación.

\section{Resultado cuantitativo}

A continuación se presentan los resultados del análisis cuantitativo. Se optó por una combinación de ambas metodologías, la cualitativa y la cuantitativa, sobre todo, con el fin de poder contrastar las ocurrencias des los mecanismos de atenuación empleados por los hablantes de cada corpus y apreciar, de este modo, las similitudes y las diferencias y, además, para poder corroborar o no la segunda hipótesis de esta investigación de que en el corpus alemán se registran más mecanismos de atenuación que en el español.

Los resultados muestran que en el corpus de las conversaciones españolas, pertenecientes al Corpus de Conversaciones Coloquiales y a las de Val.Es.Co 2.1 con un total de 47.740 palabras, se contabilizaron 522 mecanismos de atenuación y en el corpus alemán 963 mecanismos en las conversaciones correspondientes al Datenbank. für gesprochenes Deutsch, con 49.095 palabras analizadas. En el siguiente gráfico se observan los mecanismos de atenuación utilizados en cada corpus. 


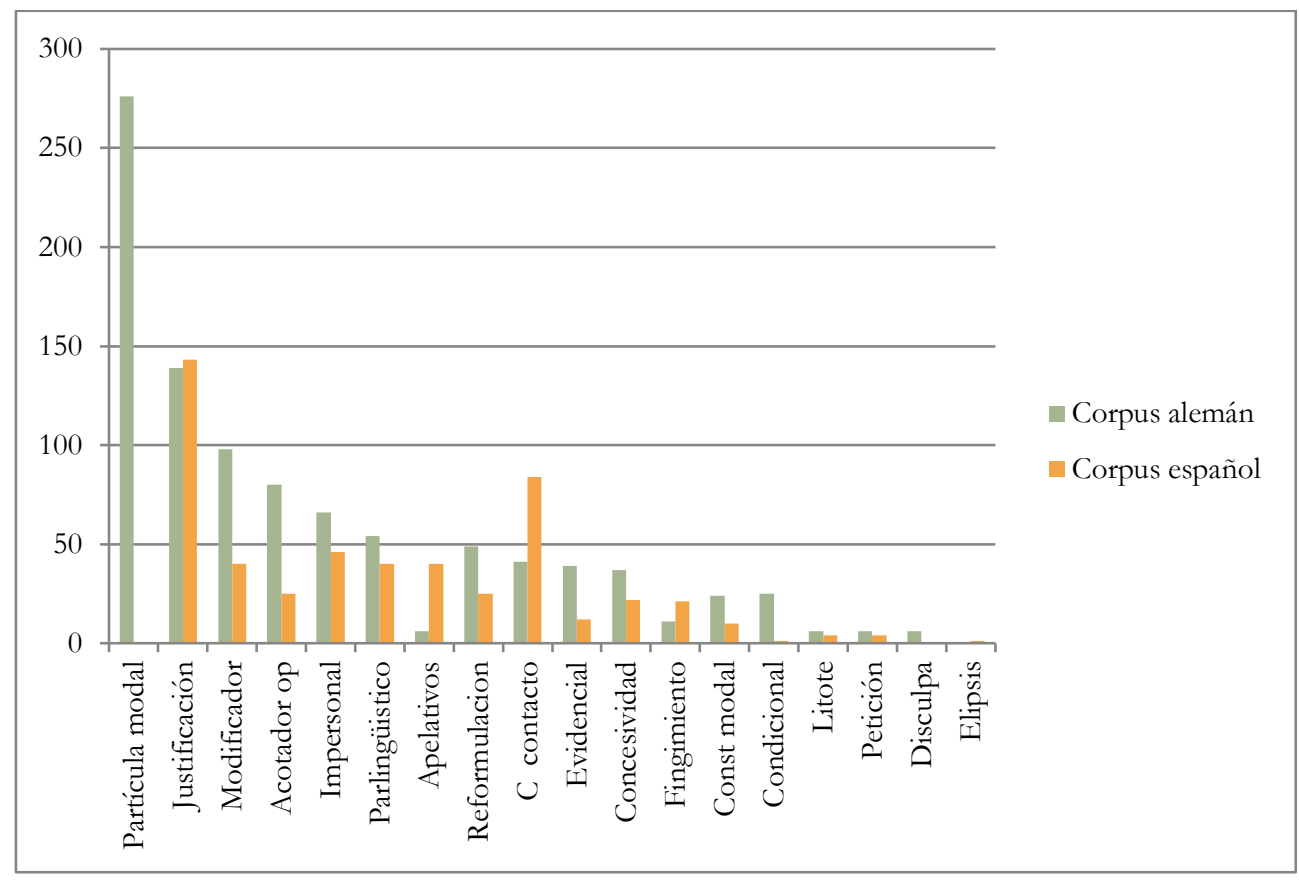

Gráfico 1. Mecanismos utilizados.

En el Gráfico 1 figuran los mecanismos utilizados en ambos corpus en números absolutos. Se aprecia que la táctica de atenuación más utilizada en el corpus alemán es la que se realiza a través de las partículas modales, en el español no aparecen estas partículas como tácticas de atenuación porque no existen en esta lengua. El segundo recurso más utilizado en ambos corpus han sido las construcciones justificadoras, pero si se observa la tabla ${ }^{8}$, se advierte una diferencia:

Tabla 1. Contraste de tácticas lingüísticas en porcentaje.

\begin{tabular}{|l|l|l|}
\hline \multicolumn{1}{|c|}{ Táctica atenuadora } & Corpus español & Corpus alemán \\
\hline Partículas modales & 0 & $28,66 \%$ \\
\hline Justificaciones & $27,39 \%$ & $14.43 \%$ \\
\hline Modificadores & $7,66 \%$ & $10,18 \%$ \\
\hline Acotador de la opinión & $4,79 \%$ & $8,31 \%$ \\
\hline Impersonalidad & $8,81 \%$ & $6,85 \%$ \\
\hline Elementos parlalingüísticos & $7,66 \%$ & $5,61 \%$ \\
\hline Fórmulas apelativas & $7,85 \%$ & $0,62 \%$ \\
\hline Reformulación & $5,36 \%$ & $5,09 \%$ \\
\hline Expresiones de control de contacto & $16,09 \%$ & $4,26 \%$ \\
\hline Evidenciales & $2,30 \%$ & $4,05 \%$ \\
\hline Movimientos concesivos & $4,21 \%$ & $3,84 \%$ \\
\hline Fingimiento & $4,02 \%$ & $1,14 \%$ \\
\hline Construcciones modales & $1,92 \%$ & $2,49 \%$ \\
\hline Usos modalizadores de tiempos verbales: Condicional & $0,19 \%$ & $2,60 \%$ \\
\hline Litote & $0,77 \%$ & $0,62 \%$ \\
\hline Petición & $0,77 \%$ & $0,62 \%$ \\
\hline Expresiones de disculpa & 0 & $0,62 \%$ \\
\hline Elipsis & $0,19 \%$ & 0 \\
\hline
\end{tabular}


Esta táctica es la más utilizada en el corpus español, 27,39\% frente a 14,43\% en el corpus alemán. En lo referente a los modificadores, si se observa el gráfico 1 y la tabla 1 , se advierte que no aparece la doble categorización entre modificadores morfológicos internos y modificadores externos, debido a que la lengua alemana no dispone de derivación morfológica con función atenuadora. En el corpus alemán llama la atención el elevado uso de construcciones acotadoras de la opinión, hecho que se explicó en el apartado de los resultados cualitativos. En lo referente a las construcciones impersonales, si bien se han contabilizado más recursos lingüísticos en el corpus alemán -66 ocurrencias frente a 46 en el corpus español-, si se observa la tabla 1, el porcentaje de este mecanismo en el corpus español es más elevado $(8,81 \%$ en español y 6,85\% en alemán). Esto es debido a que, como se ha mencionado anteriormente, los interlocutores alemanes se han servido de más recursos de atenuación, 963 frente a 522 en el corpus español, lo que lleva consigo una variación en el porcentaje. En ambos corpus los hablantes se han valido frecuentemente del recurso paralingüístico, en concreto, el de las risas, como mecanismo de atenuación. También es indicativo, y en el apartado anterior se explicó la alta frecuencia de las fórmulas apelativas, así como también partículas discursivas y las expresiones de control de contacto utilizadas en el corpus español. El uso de las construcciones que expresan fingimiento también es más elevado en el corpus español. En el corpus alemán, si bien el uso modalizador de los tiempos verbales a través del modo condicional (Konjunktiv II) no es una de las tácticas más utilizadas, se ha empleado con más frecuencia por los hablantes de esta lengua, en concreto, 25 ocurrencias frente a solamente una contabilizada en el corpus español. Asimismo, los interlocutores alemanes del corpus analizado también han hecho un mayor uso de la evidencialidad y de las construcciones con valor modal para atenuar lo dicho. En el análisis de las conversaciones alemanas se han contabilizado seis expresiones de disculpas y en el corpus español una elipsis. Esto no significa que los interlocutores no empleen estos mecanismos como tácticas de atenuación en sus discursos, sino que en el corpus analizado para esta investigación no se ha registrado ningún caso.

Si se contrastan los números totales de mecanismos de atenuación empleados por los hablantes españoles (522) y de los hablantes alemanes (963) con el número de palabras de cada corpus, a saber, 47.740 en el corpus español y 43.095 en el alemán, se advierte que por cada mil palabras los hablantes españoles se han valido de 10,9 mecanismos de atenuación frente a los 22,3 mecanismos empleados por los hablantes del corpus alemán analizado.

A modo de resumen de este apartado se aprecia que la táctica lingüística más utilizada en el corpus español son las construcciones justificadoras seguidas de las partículas discursivas y las expresiones de control de contacto, las construcciones impersonales y las fórmulas apelativas. Tanto los modificadores morfológicos internos como los elementos paralingüísticos han sido el quinto recurso más empleado por los 
interlocutores españoles. En cambio, en el corpus alemán los hablantes han hecho más uso de las partículas modales, seguidas de las construcciones justificadoras, los modificadores morfológicos externos, las construcciones acotadoras de la opinión y las construcciones impersonales. Asimismo, se ha podido observar que en el corpus analizado los hablantes alemanes han utilizado más mecanismos de atenuación que los hablantes españoles.

\section{Discusión}

El objeto de esta investigación era averiguar qué mecanismos utilizan los hablantes nativos en conversaciones coloquiales y si hay similitudes o diferencias en las tácticas utilizadas. Se partía de dos hipótesis: la segunda, donde se suponía encontrar más mecanismos de atenuación en el corpus de las conversaciones alemanas, sí se ha podido corroborar. Los hablantes alemanes del corpus analizado para este estudio han utilizado más recursos para atenuar su discurso que los interlocutores españoles, en concreto, 963 frente a 522 en conversaciones con la misma duración, a saber, 182 minutos analizados en el corpus alemán con un total de 43.095 palabras y 184 minutos correspondientes al corpus español con 47.740 palabras. Los hablantes del corpus alemán se han valido por cada mil palabras de 22,3 mecanismos de atenuación frente a los 10,9 mecanismos empleados por los hablantes españoles de nuestro corpus. En lo referente a la primera hipótesis, donde se planteaba si era de esperar que los mismos mecanismos de atenuación empleados en otros géneros se fueran a encontrar en las conversaciones coloquiales o si, por el contrario, se podía esperar que hubiera diferente uso debido a la naturaleza de las conversaciones coloquiales, se ha podido observar que aparecen con una mayor frecuencia aquellos mecanismos de atenuación más característicos del discurso oral. En el corpus español, los hablantes han utilizado modificadores morfológicos internos, fórmulas apelativas, partículas discursivas y expresiones de control de contacto, tácticas lingüísticas de atenuación más características de la conversación coloquial. Respecto al corpus alemán, se ha contabilizado con una alta frecuencia las partículas modales que se emplean, sobre todo, en el discurso oral. Las construcciones justificadoras, así como las construcciones acotadoras de la opinión y los modificadores externos son mecanismos de atenuación que pueden aparecer tanto en el discurso oral como en el escrito.

Si se comparan estos resultados con las investigaciones previas, mencionadas en la introducción de este trabajo, sobre mecanismo de atenuación en análisis contrastivos español-alemán, se observa lo siguiente: el Konjunktiv II (condicional) ha sido utilizado por los hablantes alemanes con una alta frecuencia en las tertulias televisivas donde la relación entre los interlocutores es más funcional que social y en los correos electrónicos de estudiantes a profesores con una relación jerárquica, contextos situacionales que exigen crear una mayor distancia de lo dicho para lograr el fin. En las conversaciones coloquiales alemanas del corpus analizado llama la atención la alta frecuencia, sobre todo, de las expresiones acotadoras de la expresión, mecanismo que 
se utiliza para presentar la opinión acotada al terreno de uno mismo con el fin de respetar las opiniones de los demás. En el corpus español se advierten numerosas ocurrencias con las expresiones de control de contacto y con las fórmulas apelativas que suelen tener una función afiliativa, ya que a través de estas tácticas lingüísticas se pretende conseguir una alianza con el interlocutor.

Se podría decir que las razones de estos resultados se deben, en parte, a la naturaleza de las conversaciones coloquiales y, en parte, a los parámetros socioculturales en los que se lleva a cabo cada tipo de corpus. Las partículas discursivas y expresiones de control de contacto buscan una alianza con el interlocutor y las fórmulas apelativas muestran una relación de cercanía y confianza, característica de la cultura española de afiliación y confianza (Bernal, 2007; Bravo, 1993, 2005; Hernández-Flores, 2003; Contreras, 2008, 2012) o de una ingruop-relationship (Scollon \& Scollon, 1995). En cambio, las construcciones acotadoras de la opinión están más relacionadas con el territorio y la privacidad, y la función de los modificadores externos consiste en minimizar la fuerza ilocutiva de los actos de habla y regular la relación interpersonal y social entre los interlocutores, por lo que se podría decir, que el hecho de utilizar estos mecanismos lingüísticos podría ser un reflejo sociocultural de una cultura donde prevalecen las in-outgruop-relationships (Scollon \& Scollon, 1995; Contreras, 2017). No obstante, sería interesante contrastar estos resultados con futuras investigaciones, además, hay una variable que puede haber alterado un poco los resultados obtenidos en este estudio y, creemos, que sería relevante considerarla. En las conversaciones del corpus alemán hay más hablantes femeninas que en el corpus español y para este estudio no se ha tenido en cuenta el sexo de los interlocutores, factor que podría influir en los resultados.

Para finalizar este apartado queríamos destacar las contribuciones de este estudio, tanto para el estudio de la atenuación lingüística como para la didáctica del español y del alemán como lenguas extranjeras. No existen estudios contrastivos español-alemán de mecanismos de atenuación en conversaciones coloquiales, desde nuestro conocimiento, por lo que esta investigación ha hecho patente el diferente empleo que hacen los hablantes nativos de estos mecanismos lingüísticos en conversaciones coloquiales. Estos hallazgos pueden ser esenciales para futuras investigaciones en el campo de la atenuación lingüística y en estudios didácticos.

Otra contribución interesante de los resultados obtenidos de esta investigación es que el recurso lingüístico de atenuación a través de las construcciones justificadoras se ha utilizado con una alta frecuencia en ambos corpus, tanto para salvaguardar la imagen propia, como para prevenir una posible amenaza a la imagen del interlocutor. Además, se ha visto que este mecanismo es complejo, en el sentido de que puede englobar una gran variedad de tácticas, como construcciones impersonales para 
encubrir la opinión o como recursos de parcelación de la información para retrasar lo dicho y conseguir una mayor distancia de la enunciación.

\section{CONCLUSIONES}

A modo de resumen, este estudio ha mostrado que el contexto sociocultural de los interlocutores influye en el uso de los mecanismos lingüísticos de atenuación. Por ello, hay mecanismos de atenuación que se utilizan de forma diferente, como son, en el corpus alemán, las construcciones acotadores de la opinión que presentan acotado el terreno a uno mismo, frente a las partículas discursivas y expresiones de control contacto y a las fórmulas apelativas del corpus español que están más relacionadas con la alianza y la confianza. Asimismo, se corroboran los resultados de estudios anteriores, que hay tácticas lingüísticas que no se utilizan como mecanismos de atenuación en la otra lengua, como son las partículas modales de la lengua alemana o los modificadores morfológicos internos a través del sufijo, como es el caso del diminutivo en español.

\section{REFERENCIAS BIBLIOGRÁFICAS}

Albelda, M. (2008). Atenuantes en Chile y España. Cortesía y conversación: De lo escrito a lo oral. [en línea]. Disponible en: http://www.edice.org/descargas/3coloquioEDICE.pdf

Albelda, M. (2016). Sobre la incidencia de la imagen en la atenuación pragmática. Revista Internacional de Lingüística Iberoamericana, 27, 19-32.

Albelda, M. \& Briz, A. (2010). Aspectos pragmáticos. Cortesía y atenuantes verbales en las dos orillas a través de muestras orales. En M. Aleza Izquierdo \& J. M. Enguita Utrilla (Eds.), La lengua española en América: Normas y usos actuales (pp. 237-260). Valencia: Universidad de Valencia.

Albelda, M., A. Briz, A. M. Cestero, D. Kotwica \& C. Villalba. (2014). Ficha metodológica para el análisis pragmático de la atenuación en corpus discursivos del español. (ES.POR.ATENUACIÓN). Oralia 17, 7-62.

Althaus, H.-J. \& Mog, P. (1996). Die Deutschen in ibrer Welt. Tübinger Modell einer integrativen Deutschlandkund. Berlin: Langenscheidt.

Bernal, M. (2007). Categorización sociopragmática de la cortesía y descortesía. Un estudio de la conversación coloquial española. Estocolmo: Stockholms Universitet.

Blas, J. L. (1994). Los pronombres de tratamiento y la cortesía. Revista de Filología de la Universidad de la Laguna, 13, 7-35. 
Boretti, S. (2005). ¿Actividad de imagen vs actividad de cortesía? El desarmador como estrategia interaccional. En J. Murillo (Ed.), Actas del II Coloquio del Programa EDICE (pp. 209-220). San José de Costa Rica: Universidad de Costa Rica, www.edice.org

Bravo, D. (1993). La atenuación de las divergencias mediante la risa en negociaciones españolas y suecas. Estocolmo: Universidad de Estocolmo.

Bravo, D. (2005). Categorías, tipologías y aplicaciones: hacia una redefinición de la cortesía comunicativa. En D. Bravo (Ed.), Estudios de la (des)cortesía en español. Categorias conceptuales y aplicaciones a corpora orales y escritos. (pp. 21-52). Buenos Aires: Dunken.

Briz, A. (1995). La atenuación en la conversación coloquial. Una categoría pragmática. En L. Cortés (Ed.), El español coloquial: Actas del I Simposio sobre análisis del discurso oral (pp. 103-122). Almería: Universidad de Almería.

Briz, A. (1998). El español coloquial en la conversación: Esbozo de pragmagramática. Barcelona: Ariel.

Briz, A. (2001). El español coloquial en la conversación. Esbozo de pragmática-gramática. Barcelona: Ariel.

Briz, A. (2003). La estrategia atenuadora en la conversación cotidiana española [en línea]. Disponible en: http://www.edice.org/descargas/1coloquioEDICE.pdf

Briz, A. (2007). Para un análisis semántico, pragmático y sociopragmático de la cortesía atenuadora en España y América. Lingüistica Español Actual, 29, 5-40.

Briz, A. \& Albelda, M. (2013). Una propuesta teórica y metodológica para el análisis de la atenuación lingüística en español y portugués. La base de un proyecto en común (es.por.atenuación). Onomázęin, 28, 288-319.

Briz, A. \& Grupo Val.Es.Co. (2002). Corpus de conversaciones coloquiales. Madrid: Arco Libros.

Brown, P. \& Levinson, S. (1978, 1987). Politeness. Some universals in language use. Cambridge: Cambridge University Press.

Cabedo, A. \& Uclés, G. (2019). Reparación de imagen y comportamiento prosódico: Entre la atenuación y la intensificación. Cultura, Lenguaje y Representación, 21, 23-38. https://doi.org/10.6035/CLR.2019.21.2

Caffi, C. (1999). On mitigation. Journal of Pragmatics, 3, 881-909.

Caffi, C. (2007). Mitigation. Oxford: Elsevier. 
Cestero, A. M. \& Albelda M. (2012). La atenuación lingüística como fenómeno variable. Oralia, 15, 67-124.

Contreras, J. (2004). Alternancia de turnos y cortesía. Un análisis contrastivo español/alemán. Estudios Filológicos Alemanes. Revista del Grupo de Investigación Filología Alemana, 5, 371-382.

Contreras, J. (2008). Conversational silence and face in two sociocultural contexts. Pragmatics, 18(4), 707-728.

Contreras, J. (2012). ¿Hay diferencias en las estrategias de atenuación utilizadas en los correos electrónicos españoles y alemanes? Oralia, 15, 325-42.

Contreras, J. (2017) Intensivierungs- und Abschwächungsmechanismen in Talkshows: Eine Analyse Spanisch-Deutsch. En C. Ehrhardt \& E. Neuland (Eds.), Sprachliche Höflichkeit. Historische, aktuelle und künftige Perspektiven (pp. 207-222). Tübingen: Narr Francke Attempto.

Contreras, J. (2018). La impersonalidad como estrategia de atenuación en la novela El lector de Julio Verne de Almudena Grandes: Un análisis contrastivo alemán/español. Nuevas aportaciones sobre la atenuación pragmática. Formas, funciones y variación lingüistica Rilce. Revista de Filología Hispánica, 34(3), 1243-1258.

Contreras, J. \& Zhao L. (2017). Análisis contrastivo de estrategias de atenuación en un corpus de correos electrónicos españoles, alemanes y chinos. En M. Albelda \& W. Mihatsch (Eds.), Atenuación e intensificación en géneros discursivos: Iberoamericana, 65 (pp. 205-220). Madrid: Iberoamericana.

De Miguel, A. (1997). Autobiografía de los españoles. Como nos vemos. ¿Somos así? Barcelona: Planeta.

Figueras, C. (2017). Atenuación y construcción de la identidad de experto en trastornos de la conducta alimentaria. En M. Albelda \& W. Mihatsch (Eds.), Atenuación e intensificación en géneros discursivos (pp. 169-186). Iberoamericana, 65. Madrid: Iberoamericana.

Fraser, B. (1980). Conversational mitigation. Journal of Pragmatics, 4, 341-50.

Goffman, E. (1967). Interactional ritual: Essays face-to-face behaviour. Nueva York: Pantheon Books.

Haverkate, H. (2004). El análisis de la cortesía comunicativa: Categorización pragmalingüísitca de la cultura española. En D. Bravo \& A. Briz (Eds.), Pragmática sociocultural: Estudios sobre el discurso de cortesía en español (pp. 55-65). Barcelona: Ariel. 
Hernández-Flores, N. (2003). Cortesía y contextos socioculturales en la conversación española de familiares $y$ amigos [en línea]. Disponible en: http://www.edice.org/descargas/1coloquioEDICE.pdf

Hernández-Flores, N. (2008). Politeness and other types of facework: Communicative and social meaning in a television panel discussion. Pragmatics, 18(4,) 577-603.

Hernández-Flores, N. (2013). Actividad de imagen. Caracterización y tipología en la interacción comunicativa. Pragmática Sociocultural, 1(2), 1-24.

Holly, W. (1979). Imagearbeit in Gesprächen zur linguistischen Beschreibung des Beziehungsaspekts. Tübingen: Max Niemeyer Verlag.

Kaul de Marlangeon, S. (2008). Tipología del comportamiento verbal descortés en español. En A. Briz Gómez, A. Hidalgo Navarro, M. Albelda Marco, J. Contreras Fernández \& N. Hernández Flores (Eds.), Cortesía y conversación: De lo escrito a lo oral. Tercer Coloquio Internacional del Programa EDICE (pp. 254-266). Valencia/Estocolmo: Universidad de Valencia-Programa EDICE.

Kotwica, D. (2018). Verbos de percepción evidenciales en artículos científicos del siglo XIX: funciones pragmático-retóricas. Nuevas aportaciones sobre la atenuación pragmática. Formas, funciones y variación lingüistica RILCE: Revista de Filología Hispánica, 34(3), 1154-1178.

Lakoff, G. (1972). Hedges: A study in meaning criteria and the logic of fuzzy concepts. Journal of Philosophical Logics, 2, 458-508.

Langner, M. (1994). Zur kommunikativen Funktion von Abschwächung. Pragma- und soziolinguistische Untersuchungen. Münster: Nodus Publikationen.

Luukka, M.-R. \& Markkanen R. (1997). Impersonalization as a form of hedging. En R. Markkanen \& H. Schröder (Eds.), Research in Text Theory: Hedging and Discourse: Approaches to the Analysis of a Pragmatic Phenomenon in Academic Texts (pp. 168187). Berlin/Boston: De Gruyter.

Mihatsch, W. (2013). Hedges. The enciclopedia of applied linguistics, 2457-2462.

Samper Hernández, M. (2018). Un cambio en tiempo real: La atenuación entre hablantes universitarios de Las Palmas de Gran Canaria. Nuevas aportaciones sobre la atenuación pragmática. Formas, funciones y variación lingüistica RILCE: Revista de Filología Hispánica, 34(3), 1259-1279.

Sifianou, M. (2012). Disagreements, face and politeness. Journal of Pragmatics, 44, 15541564. 
Scollon, R. \& Scollon, S. W. (1995). Intercultural communication. A discourse approach. Cambridge: Cambridge University Press.

Soler Bonafont, M. A. (2018). Fingimientos y atenuación en el uso de creo. Nuevas aportaciones sobre la atenuación pragmática. Formas, funciones y variación lingüística RILCE: Revista de Filología Hispánica, 34(3), 1104-1128.

Uclés Ramada, G. (2018). La atenuación de los marcadores de control de contacto en PRESEEA: Un estudio comparativo entre España y México. Nuevas aportaciones sobre la atenuación pragmática. Formas, funciones y variación lingüística RILCE: Revista de Filología Hispánica, 34(3), 1313-1335.

Villalba, C. (2018). Primera persona del plural en los juicios orales. Valor representativo y estrategia atenuante. Nuevas aportaciones sobre la atenuación pragmática. Formas, funciones y variación lingüistica RILCE: Revista de Filología Hispánica, 34(3), 1056-1080.

Waltereit, R. (2006). Zur Pragmatik und historischen Semantik von Modalpartikeln und ibren funktionalen Äquivalenten in romanischen Sprachen. Tübingen: Niemeyer.

\section{NOTAS}

1 Esta investigación se enmarca dentro del Proyecto ES.VAG.ATENUACIÓN ("La atenuación pragmática en su variación genérica: géneros discursivos escritos y orales en el español de España y América” MINECO FFI2016-75249-P).

${ }^{2}$ Se utilizara indistintamente mecanismo, recurso o táctica (lingüística).

3 Si bien Cabedo y Uclés (2019) se plantean si la función reparadora de la imagen se lleva a cabo mediante la atenuación o la intensificación fónica, nosotros en este trabajo nos basamos en Albelda et al. (2014) y, por lo tanto, en su función atenuadora.

${ }^{4}$ Médicos/as, enfermeros/as e ingenieros/as.

${ }^{5}$ A partir de ahora se citará Albelda et al. (2014).

${ }^{6} \mathrm{La}$ autora de esta investigación ha traducido y ha puesto en cursiva los ejemplos justo debajo de los ejemplos alemanes, a excepción de los ejemplos (10), (11) y (12) donde se ha insertado la traducción dentro de la explicación del mecanismo lingüístico de atenuación.

${ }^{7}$ No aparece todo el fragmento, pues aparecen secuencias laterales y solapamientos, pero la persona que habla es la misma y sigue con su turno. 
${ }^{8}$ Los mecanismos de atenuación están basados en las tácticas lingüísticas de la ficha propuesta por Albelda et al. (2014), excepto las partículas modales que no figuran en dicha ficha por no existir en la lengua española. 